\title{
A WCS-BASED APPROACH TO INTEGRATE SATELLITE IMAGERY DATA IN WILDFIRE SIMULATION
}

\author{
António Esteves ${ }^{1}$ and António Pina ${ }^{1}$ \\ ${ }^{1}$ Departamento de Informática, Universidade do Minho, Braga, Portugal \\ \{esteves,pina\}@di.uminho.pt
}

\begin{abstract}
Keywords: $\quad$ OGC standards, WCS, Satellite datasets, MODIS sensor, Wildfire simulation.
Abstract: $\quad$ This paper describes the integration of multi-dimensional data from satellite sensors in a Civil Protection application that simulates fire spread. The approach uses standard Web Coverage Services from OGC to fetch and process land cover and recently burned areas, available in the form of satellite imagery data previously captured by the MODIS sensor, to automatically generate renovated fuel maps. The proposed architecture is based on rasdaman, a domain-independent database management system (DBMS) that offers a suite of WCS services on top of the DBMS. In the current work we extended rasdaman with facilities to: (i) insert and retrieve multi-layer coverages from WCS, (ii) support new formats, such as HDF, adequate for satellite imagery and multi-layer files, and (iii) support Coordinate Reference Systems. We also demonstrate that it is feasible to use MODIS datasets to automatically compute valuable and regularly updated fuel maps, used as input of fire spread simulations. The results also show that in spite of using inexpensive general and low resolution $(500 \mathrm{~m})$ MODIS maps, we obtained quite acceptable results when compared with the static ones, which are tailored and higher resolution $(80 \mathrm{~m})$.
\end{abstract}

\section{INTRODUCTION}

This work is part of the Portuguese CROSS-Fire project aimed to develop a grid-based risk management decision support system, for the Civil Protection $(\mathrm{CP})$ authorities, using wildfires as the main case study, and FireStation (Lopes et al., 2002) a simulator to compute the fire spread over a complex topography.

The paper reports the work that has been carried on to improve the quality of fire spread simulations by automatically renewing the old static fuel maps used as input, with fresh data obtained from satellite sensors, as an alternative to a time and money consuming human update. The approach uses standard Web Coverage Services (WCS) from Open Geospatial Consortium (OGC) to get and process land covers and recently burned areas, available in the form of satellite imagery data, to automatically generate renovated fuel maps.

To integrate information models, encodings, and metadata we use: i) a central core of Web services, implemented as a set of Web Processing Service (WPS) algorithms, ii) the EGEE/EGI to provide raw technological capability provision, iii) a Spatial Data Infrastructure (SDI), to provide the access and management of remote geospatial data from remote sen- sors/satellites or in-situ sensors, and iv) a GUI that provides users with visualization facilities, and metadata and spatial data queries (Pina et al., 2011).

The overall software development take advantage of available implementations of the standards proposed by the OGC: (i) the OGC Web Services (OGCWS), (ii) the OGC Sensor Web Enablement (OGCSWE) services, and (iii) gvSIG, a full-featured Open Source GIS desktop GUI.

The simulator requires both static and dynamic input data. The static data comprises: i) the terrain divided into cells, each one characterized by its altitude and fuel type, ii) the wind conditions, and iii) the control parameters. The dynamic data, is provided by a Remote Weather Station Sensor Observation Service (SOS) client. Given the potential of the imagery data that may be obtained from satellite sensors we evaluated the possibility of computing improved and updated fuel maps based on: i) updated vegetation, ii) newly burned areas, and iii) land cover type, by getting and processing data from MODIS (USGS, 2008) sensors aboard of Terra and Aqua satellites. To maintain the commitment to OGC standards we use the WCS (Whiteside and Evans, 2008) and its Web Coverage Processing Service (WCPS) (Baumann, 2009) extension. 
Among the related work we should mention the SAFORAH project, the work by Daniel Mandl at NASA, Sentinel/Digital Asia, GEO Grid, and RGIproject projects, and the tools developed by NASA to process and deliver data from MODIS/Aster sensors: the MODIS Rapid Response system and Fire Information for Resource Management System.

\section{OGC STANDARDS}

The WCS standard specifies 3 operations: GetCapabilities, DescribeCoverage, and GetCoverage. These operations allow spatial, temporal and band subsetting, scaling, reprojection, and format encoding.

The WCS-Transactional (WCS-T) extension to WCS 2.0 allows the insertion and updating of coverages stored on a WCS server. WCS-T specifies an additional Transaction operation.

The Web Coverage Processing Service (WCPS) extension to WCS 2.0 defines a flexible interface for the navigation, extraction, and ad-hoc analysis of large, multi-dimensional raster coverages. WCPS is abstract in that it does not anticipate any particular protocol, and specifies an additional ProcessCoverages operation.

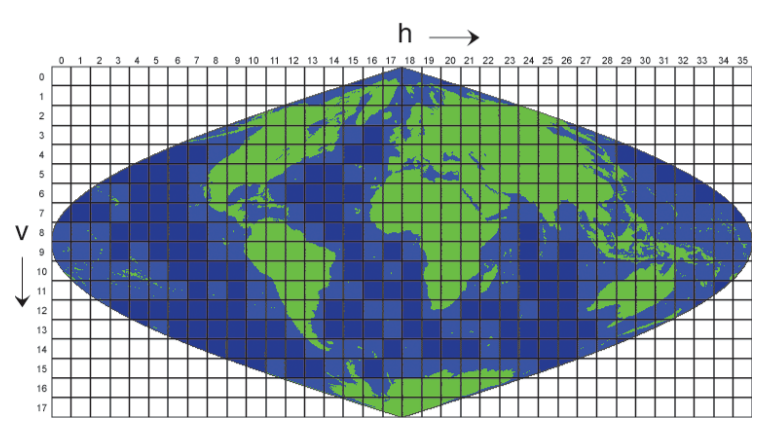

Figure 1: MODIS tiling scheme.

\section{MODIS SENSOR}

MODIS sensor is placed aboard of Terra and Aqua satellites, both covering the entire surface of the planet Earth. The planet is divided into 36X8 tiles, and the Portuguese continental land and islands spread over 3 tiles: h16v05, h17v04 and h17v05. The spatial resolution of MODIS data can be $250 \mathrm{~m}$, $500 \mathrm{~m}$ or $1000 \mathrm{~m}$ and classified in 3 categories: atmospheric, land cover, and oceanographic data. NASA provides this information at several levels, L0 up to L4, where L0 is raw data and L4 are the most processed products. MODIS L2 to L4 products are de- fined on a global sinusoidal grid. The grid is divided into fixed-area tiles of approximately 10x10 degrees in size. Each tile is assigned a horizontal (h) and vertical (v) coordinate ranging from 0 to 35 and 0 to 17 , respectively (figure 1) (Boschetti et al., 2009).

Produced from daily surface reflectance, MCD45A1 is a monthly L3 500m product that approximates the date of burning, and maps the spatial extent of recent fires. MCD12Q1 L3 product describes land cover properties derived from one year of observations. It is delivered yearly at $500 \mathrm{~m}$ resolution and incorporates 5 different land cover classification schemes: IGBP, UMD, LAI/FPAR, NPP, and PFT. Using MODIS reflectances, vegetation indices are computed daily. Difference Vegetation Index (NDVI) and Enhanced Vegetation Index (EVI) are among these indices. MOD13Q1 is a L3 grid product delivered every 16 days at $500 \mathrm{~m}$ spatial resolution. In the present work, we are interested in the level-3 (L3) land products described in table 1 and selected the IGBP scheme since it is the most detailed and comprehends 17 classes of land cover type.

Table 1: MODIS land products.

\begin{tabular}{|c|c|c|c|}
\hline $\begin{array}{c}\text { Product } \\
\text { Info. }\end{array}$ & $\begin{array}{c}\text { Product } \\
\text { Code }\end{array}$ & $\begin{array}{c}\text { Resol. } \\
(\mathrm{m})\end{array}$ & $\begin{array}{c}\text { Temporal } \\
\text { Granularity }\end{array}$ \\
\hline Burned Area & MCD45A1 & 500 & Monthly \\
Veg. Indices & MOD13Q1 & 250 & 16 Day \\
Land Cover & MCD12Q1 & 500 & Yearly \\
\hline
\end{tabular}

\section{ARCHITECTURE}

The architecture that integrates the data provided by MODIS on the fire spread simulator is supported by the following technologies: the Hierarchical Data Format (HDF) used by NASA to publish MODIS data, the Java HDF Object Package that provides an object-oriented interface to HDF data objects (The_HDF_Group, 2010), and rasdaman ((rasdaman $\mathrm{GmbH}), 2011)$, which is a domainindependent database management system (DBMS) with a WCS suite of services built on top of it. From bottom to top, the architecture (figure 2) includes:

- The postgreSQL relational DBMS that manages and stores the coverages and their metadata in two separated databases;

- The core modules of rasdaman that mediate the interaction between the WCS services (petascope) and the DBMS, and the format converters; 


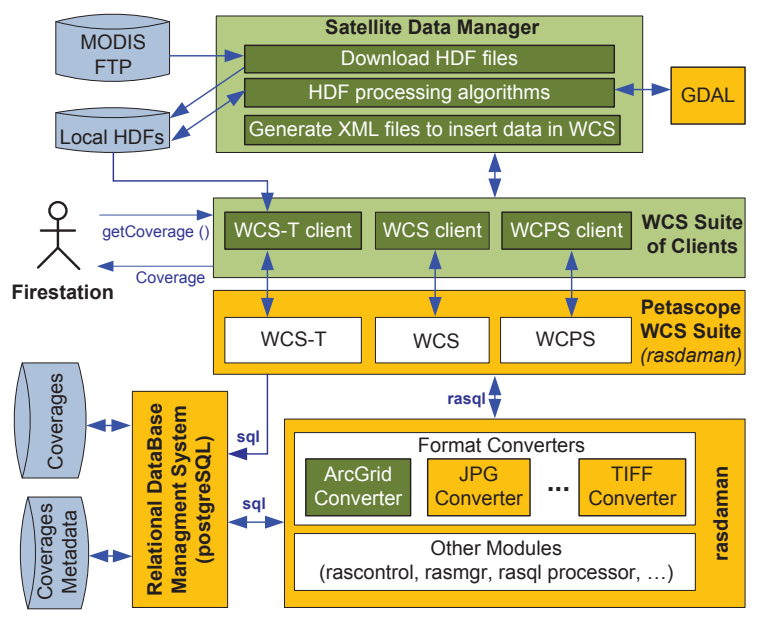

Figure 2: Proposed architecture, based on rasdaman, to extend the fire spread simulator with satellite data.

- petascope implements a OGC-compliant WCS 2.0 suite of services: WCS, WCPS, and WCS-T;

- The WCS suite of clients implements the clients that dialogue with petascope WCS services, generating the adequate XML requests and parsing the XML answers from the WCS servers;

- The Satellite data manager provides: i) a module to download HDF files from the MODIS site to a local directory; ii) a collection of algorithms to process the HDF files; iii) a module to generate the XML files necessary to insert datasets and their metadata in WCS.

The developed HDF processing algorithms permit to: (1) generate datasets for tiles missing on MODIS site (full ocean tiles), (2) extract a dataset from an HDF, (3) merge datasets when the requested region spans more than one tile, (4) reproject a dataset in order to combine datasets that use different projections, (5) extract a subset from a dataset, (6) modify the resolution of a dataset for combining/comparing datasets with different resolutions, (7) modify the range of a dataset, (8) compare datasets, and (9) combine datasets in a fuel map. Comparing datasets is needed, for example, to validate if a dynamic land cover scheme may replace a static scheme (figure 3 ). The algorithm that combines a land cover dataset with one or more burned area datasets, in order to update the yearly produced land cover with the monthly produced burned areas, is summarized in figure 4 .

\section{RESULTS}

Initially, we compared the static and the MODISbased land cover classification schemes, by previ-

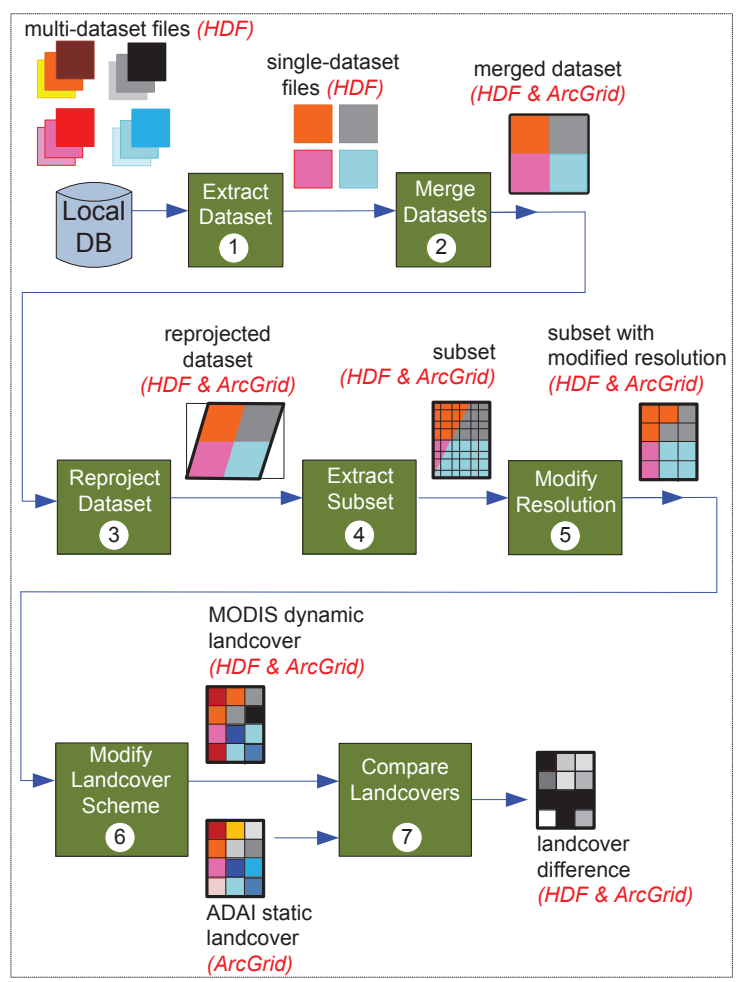

Figure 3: Processing flow to compare static (ADAI) land cover scheme with MODIS-based scheme.

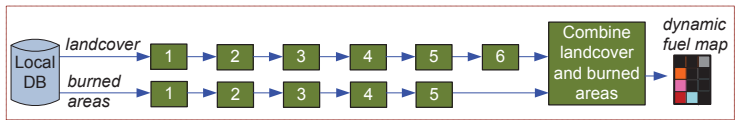

Figure 4: Processing flow to compute a MODIS-based dynamic fuel map.

ously replacing each class of the MODIS map by its static $\left(\mathrm{ADAI}^{1}\right)^{2}$ equivalent, to be able to calculate the difference between the maps. Based on the results from this comparison, a mapping from static to MODIS land cover classification scheme was elaborated (Esteves, 2011). To quantify the quality of the mapping MODIS $\mapsto$ static land cover schemes, we measured the difference among the static classes, considering 6 fuel characteristics of each class: fuel loading dead after 1 hour/10 hours/100 hours, fuel loading live, heat content, and moisture content of extinction (Cruz, 2005). Subsequently, we estimated the maximum value of the difference for each characteristic (1 to 6), among all land cover classes (0 to 16). Finally, the global difference between two static land cover classes was computed. Figure 5 displays the results of comparing MODIS-based with static land cover map. Black colour pixels represent a perfect

\footnotetext{
${ }^{1}$ ADAI - Associação para o Desenvolvimento da Aerodinâmica Industrial, Coimbra, Portugal, www.adai.pt.
} 
match ( $0 \%$ difference) between maps and a lighter colour means a greater difference. The mean difference is $14 \%$ and the average difference is $2 \%$.

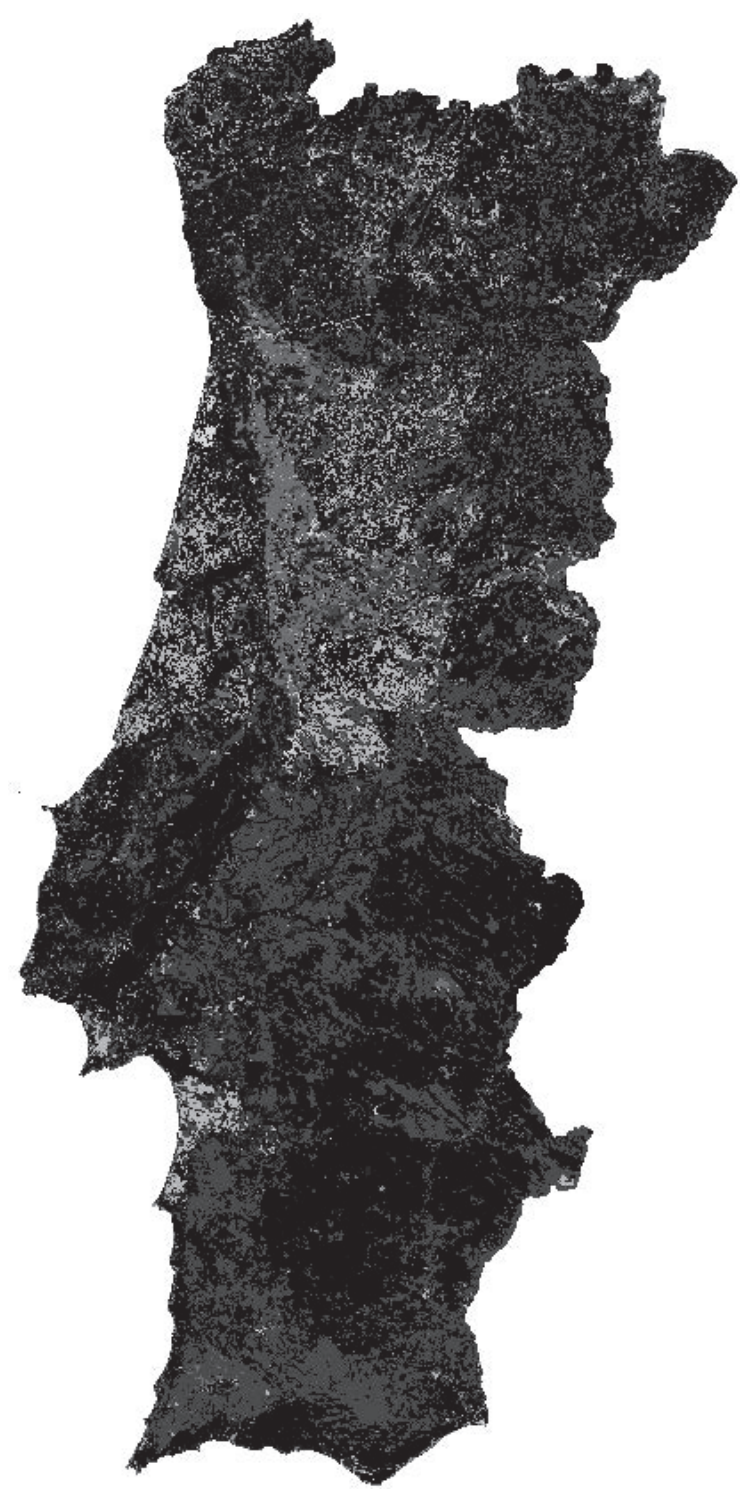

Figure 5: Difference between the MODIS-based and static land cover maps for Portuguese land.

\section{CONCLUSIONS}

rasdaman is a running project still lacking some facilities. In the course of this work, we made several extensions, including: i) insertion and retrieving of multi-layer coverages from WCS, ii) support for formats, such as HDF, for satellite imagery and for multi-layer files, and iii) support to Coordinate Ref- erence Systems. It is well known that MODIS resolution is moderate. However, our results prove that it may be used to improve the quality of static fuel maps, by combining fresh burned areas information with the land cover datasets, and to feed Firestation with the generated valuable fuel. These maps may be further improved with vegetation data produced more frequently. In the future, we plan to evaluate the architecture with higher resolution sensors/satellites, such as ASTER/Terra, ETM+/Landsat-7, SAR/ESR2 and ASAR/Envisat, PROBA, SPOT-4/5, AVNIR2/ALOS, or DEIMOS-1. We also plan to contribute, with others academics, industrial institutions and Portuguese Civil Protection, to create a national platform for fast and reliable wildfire management, based in a distributed computing infrastructure built on top of the EGI/Grid middleware.

\section{REFERENCES}

Baumann, P. (2009). The OGC Web Coverage Processing Service (WCPS) Standard. Geoinformatica.

Boschetti, L., Roy, D., and Hoffmann, A. (2009). MODIS Collection 5 Burned Area Product - MCD45, User's Guide version 2.0.

Cruz, M. G. (2005). Guia fotográfico para identificação de combustíveis florestais - Região Centro de Portugal. Centro de Estudos sobre Incêndios Florestais - ADAI, Coimbra.

Esteves, A. (2011). A wcs-based integration of multidimensional data on firestation grid application. Technical report, Departamento de Informática, Universidade do Minho, Portugal. https://pop.cp.di.uminho.pt/crossfire/wp-content/uploads/2011/11/PaperSatelliteInfo_a4.pdf.

Lopes, A., Cruz, M., and Viegas, D. (2002). FireStation an integrated software system for the numerical simulation of fire spread on complex topography. Environmental Modelling and Software, (17(3):269285).

Pina, A., Esteves, A., Puga, J., and Oliveira, V. (2011). A Geographical Information System for wild fire management. Proceedings of the 5th Iberian Grid Infrastructure Conference (Ibergrid'2011), pages 144-155.

(rasdaman $\mathrm{GmbH}$ ), P. B. (2011). The Rasdaman Project Documentation. http://kahlua.eecs.jacobsuniversity.de/trac/rasdaman.

The_HDF_Group (2010). The HDF Object Package. http://www.hdfgroup.org/hdf-java-html/hdf-object.

Whiteside, A. and Evans, J. (2008). Web Coverage Service (WCS) Implementation Standard, Open Geospatial Consortium Document 07-067r5. 